\title{
Design of Temperature Control System for Electric Heating Furnace Based on C51 Microcontroller
}

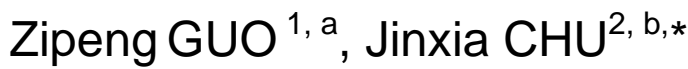 \\ ${ }^{1}$ Automobile Institute, Hubei Polytechnic Institute, Xiaogan 432000, China; \\ ${ }^{2}$ School of Fine Art \& Design, Hubei Engineering University, Xiaogan 432000, China \\ a 409461117 @qq.com, b346917126@qq.com \\ *Corresponding author
}

\section{Keywords: C51 Microcontroller; Temperature Controlling; PWM; Sensors Array}

\begin{abstract}
Accurate and rapid control of the temperature of electric heating furnace in industrial production has important practical significance and application value. In this paper, the electric heating furnace temperature control system is designed based on C51 microcontroller, equipped with temperature sensors, AD conversion module and display module. The PWM control signal, generated by the microcontroller, is used to controlling the working time of the heating resistance wire and the average power output of the resistance wire. the incremental PID algorithm is adopted to realize the temperature control of the electric heating furnace. Experiments show that: in the $0-500 \mathrm{C}^{\circ}$ range, electric heating furnace temperature control time is 60 s, temperature control relative error of up to $1 \%$. The system has the characteristics of low hardware cost, high temperature control accuracy, good reliability and strong anti - interference ability.
\end{abstract}

\section{Introduction}

With the development of science and technology and industrial production level, the electric heating furnace has been widely used in metallurgy, chemical, machinery and other kinds of industrial control, and occupies a pivotal position in the national economy. For such a nonlinear, large delay, large inertia, time-varying and unidirectional rising control object [1,3], it is difficult to establish accurate mathematical model, so the traditional control theory and methods depend on the precise mathematical model is difficult to achieve good ccontrol effect [4-7].

Based on the C51 MCU , temperature control system of electric heating furnace is designed and the classic PID temperature control algorithm, adopted in this system, and the temperature control accuracy reached higher.

\section{The design for the Whole System}

The system structure diagram is shown in Figure 1, the system consists of C51 microcontroller, temperature control circuit, electric heater, sensor array, amplifying and filtering circuit, a multi-channel switch, ADC module and LCD display module. The C51 microcontroller is ordinary 80C51 microcontroller for the control of the entire system, the acquisition of sensor signals and electrical heating control algorithm. The temperature control drive circuit is the bridge between the electric heating furnace and the controller. The sensor array is composed of a plurality of temperature sensors which are distributed in different places of the heating furnace so as to realize the accurate acquisition of the temperature of the whole heating furnace. The amplifier and filter circuit is used to amplify and filter the interference signal. The ADC module is used to collect the real-time temperature data of each sensor.

The input terminal of the bridge circuit to measure the furnace temperature by the thermal resistance temperature measuring element and a resistance element and converted into voltage signal to the amplifier, the signal into a $0-5 \mathrm{~V}$ voltage signal, the filter is sent to the multiplexer switch CD4051, multi-channel signal into the A/D converter, finally by MCU control the acquisition, SCM 
the digital quantity through digital filtering, scale conversion, on the one hand, LED will display the temperature; on the other hand, the value of the temperature value and the measured temperature, according to the deviation of the size of the proportional differential control (PID control), the size of the heating power solid state relay temperature control circuit and electric wire. The temperature of the control circuit, which tends to be given value and balance.

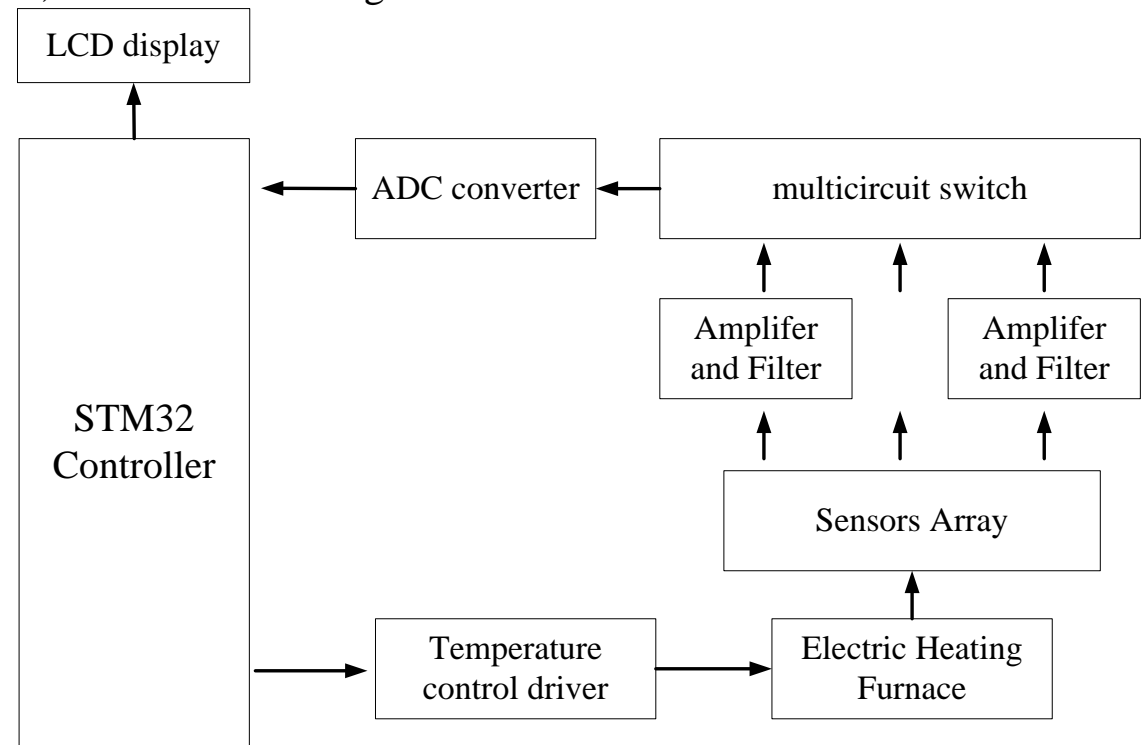

Fig. 1 diagram for the Temperature control system

\section{The Hardware Design for Designed System}

The Sensor Sampling Circuit. The sensor sampling circuit is shown in Figure 2, the sampling circuit is composed of amplifier circuit and the proportion of the Huygens bridge. The sensor uses the Pt resistance wire, in the 0 to 500 DEG C measuring range, its resistance change is 100-280.9. the resistance change can be sampled by The bridge circuit composed of $\mathrm{R} 4$, $\mathrm{R} 5, \mathrm{R} 7$, and then sent to signal amplification through proportion amplifying circuit composed of R8, R6, R3, R9 and LM324. After amplification of 20 times, the temperature signal can be input to the next level of processing circuit.

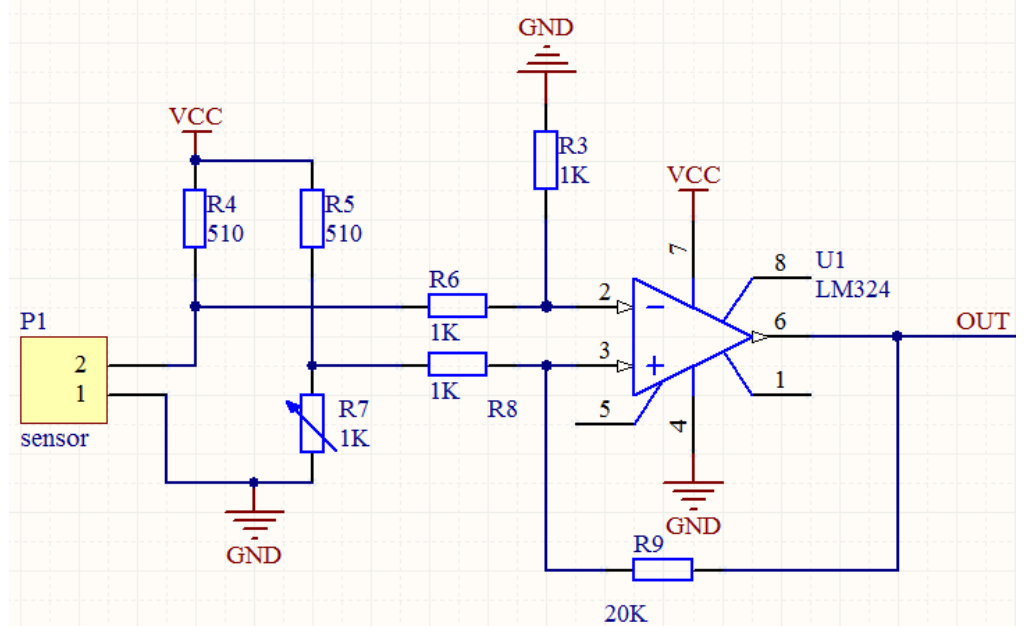

Fig. 2 temperature sampling circuit

The Sampling and Control Circuit for ADC Peripheral. The A/D converter AD574, which is produced by AD 12 bit approximation A/D conversion chip, is adopted in this design. In general, without any external circuit, it can achieve 12 bit A/D conversion, with the $+5 \mathrm{~V}$ and $-15 \mathrm{~V}$ power supply and by coupled with analog input, given the start conversion signal. At the end of the A/D conversion, the A/D conversion chip will output the conversion end signal and read the conversion data through CPU. 


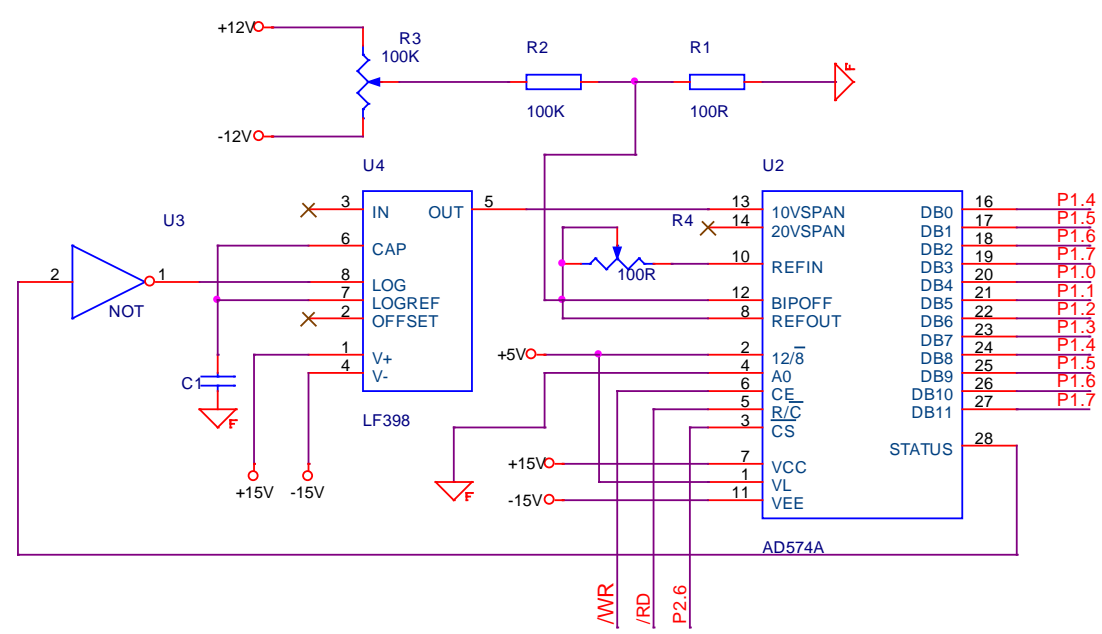

Fig. 3 the sampling and control circuit for ADC Peripheral

The AD conversion circuit is shown in Figure 3, the analog voltage signal input into this circuits through the 3th foot of LF398, and output to AD574 through 5th foot, after voltage conversion by AD574, the microcontroller can read the converted signal in real time through DB0-DB11 of the converter AD574.

Driving circuit for temperature control. The temperature control circuit is shown in Figure 4, the circuit is controlled by a single chip AC solid-state relay to adjust the load power to achieve the purpose of regulating the temperature. Z type AC solid state relay SSR is used to realize zero trigger AC power regulation. SSR is equipped with a photoelectric isolation circuit, which can reduce the mutual interference between the grid and the power grid, which is a more advanced control method. Through set P2.7 port as high level for MCU and then set as low level, you can get a positive pulse and the pulse is used to trigger the 555 after inverting the chip, resulting in a cycle of sine wave in the output of 555, then added to the heating wires. The MCU controls the heating power of the electric stove wire by controlling the duty cycle of the output pulse.

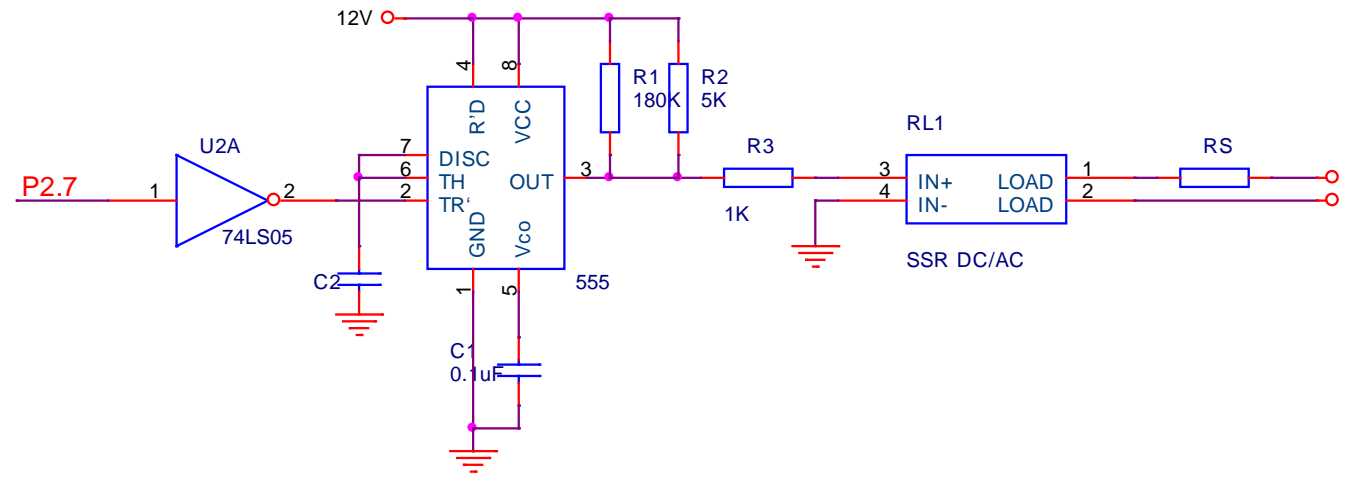

Fig. 4 Driving circuit for temperature control

\section{The Flow Chart for the Designed Software}

After power on, the system is initialized first, initialization clock, timer, interrupt and IO peripherals, then the system enters the circular wait state. once the interrupt occurs, the microcontroller began to sample temperature, and then digital filtering, nonlinear correction in MCU inside. At last, the measured temperature can be calculated, and temperature error can be achieved compared with the current temperature, and then the MCU execute the implementation of PID control algorithm. When the temperature reaches the set value, then enter into wait state, otherwise, continue to carry out temperature sampling, the PID algorithm is implemented until the temperature reaches the set value within the allowable error range. 


\section{Conclusions}

The temperature control of the electric heating furnace is designed and realized by using the C51 single chip microcomputer, with the sensor array, the temperature control driving circuit, the amplifying and filtering circuit, the sampling circuit of the sensor and so on. The experimental results show that the temperature control precision of the whole system is very high and stable.

\section{Acknowledgement}

We would like to express our thanks to ours students for their valuable discussions and helps to ready for ours experiment. This work has been supported by the key project of Hubei Provincial Department of Education: D20152703.

\section{References}

[1] Wittwer C T, Ririe K M, Andrew R V, et al. The LightCyclerTM: a microvolume multisample fluorimeter with rapid temperature control[J]. Biotechniques, 1997, 22(1): 176-181.

[2] Soltman D, Subramanian V. Inkjet-printed line morphologies and temperature control of the coffee ring effect[J]. Langmuir, 2008, 24(5): 2224-2231.

[3] Abilov A G, Zeybek Z, Tuzunalp O, et al. Fuzzy temperature control of industrial refineries furnaces through combined feedforward/feedback multivariable cascade systems[J]. Chemical Engineering and Processing: Process Intensification, 2002, 41(1): 87-98.

[4] Lin C J. A GA-based neural fuzzy system for temperature control[J]. Fuzzy Sets and Systems, 2004, 143(2): 311-333.

[5] Soyguder S, Alli H. An expert system for the humidity and temperature control in HVAC systems using ANFIS and optimization with Fuzzy Modeling Approach[J]. Energy and Buildings, 2009, 41(8): 814-822.

[6] Liying Z, Guoshu Z. Application of fuzzy-PID control algorithm in uniform velocity temperature control system of resistance furnace[J]. Chinese Journal of Scientific Instrument, 2008, 29(2): 405.

[7] Chen Y, Lei J, Yang X. Variable Discourse of Universe Fuzzy-PID Temperature Control System for Vacuum Smelting Based on PLC[C]. 2009 WRI Global Congress on Intelligent Systems. IEEE, 2009, 1: 541-544.

[8[ Soyguder S, Alli H. An expert system for the humidity and temperature control in HVAC systems using ANFIS and optimization with Fuzzy Modeling Approach[J]. Energy and Buildings, 2009, 41(8): 814-822.

[9] Roper M G, Easley C J, Legendre L A, et al. Infrared temperature control system for a completely noncontact polymerase chain reaction in microfluidic chips[J]. Analytical chemistry, 2007, 79(4): 1294-1300. 